\title{
Endoscopic Resection of a Large Pedunculated Colonic Polyp Using an Insulated-Tip Diathermy Knife
}

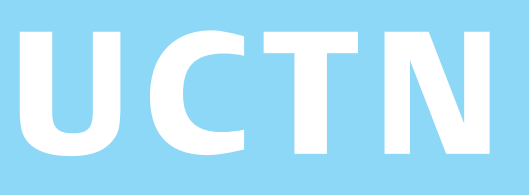

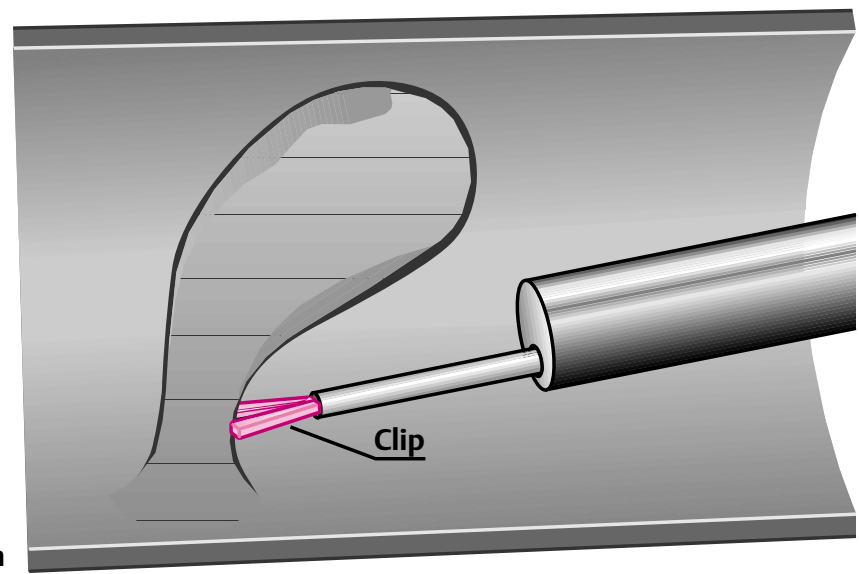

a

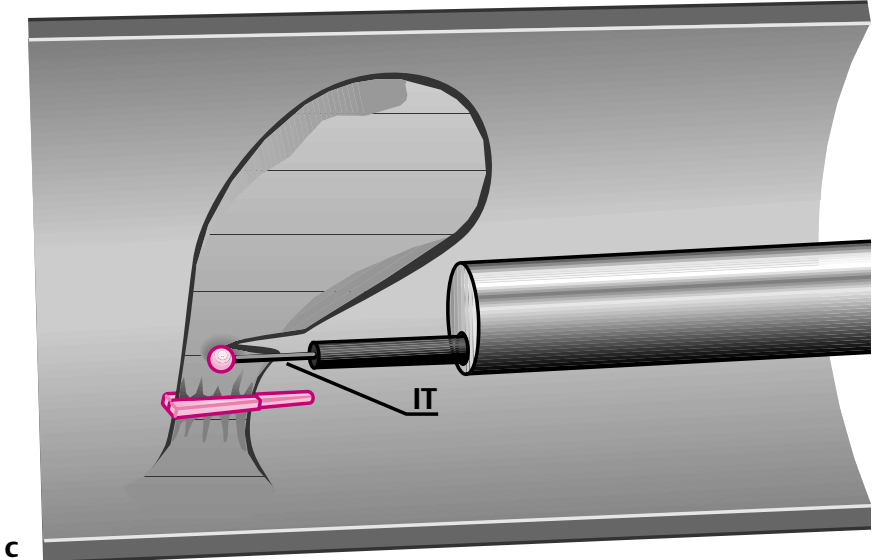

Figure 1 Schematic showing endoscopic resection of a polyp using an insulated-tip diathermy knife. a A long metal clip is opened and applied to the stalk of the polyp, close to the colon wall. $\mathbf{b}$ The clip

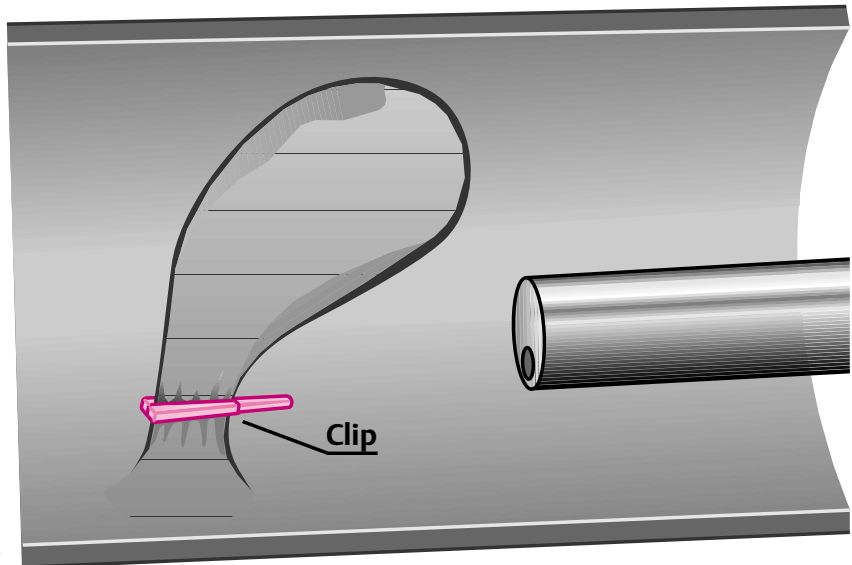

b

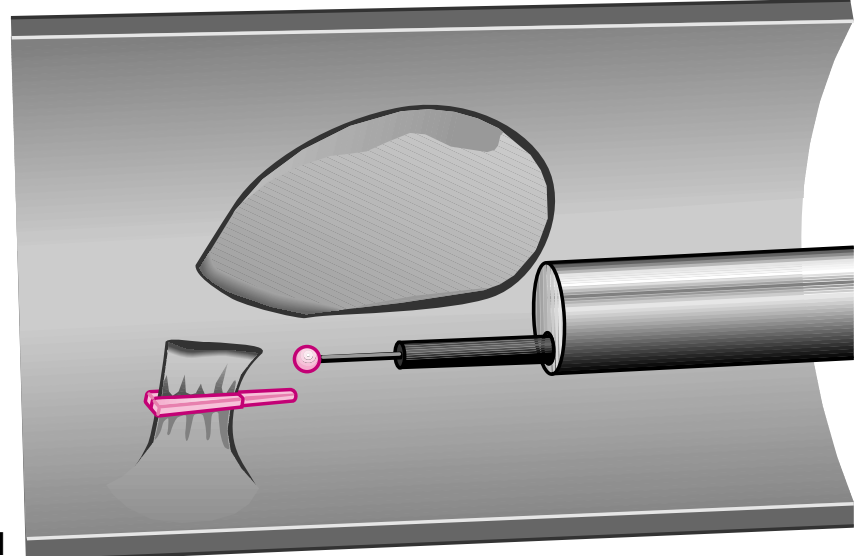

clamps the stalk. c The stalk above the clip is cut with an insulated-tip diathermy knife (IT). d The lesion is removed from.
A large pedunculated polyp was discovered incidentally during colonoscopic examination in a 70-year-old Japanese woman. She had undergone the examination after a positive fecal occult blood test. The polyp was located in the sigmoid colon and had a long stalk and a large head. Its removal was considered to be technically demanding because the head of the polyp nearly occluded the lumen of the colon, making it difficult to maneuver a snare over the polyp. The stalk of the polyp was fully visible, however, and we attempted endoscopic resection using an insulated-tip diathermy knife (KD-610L; Olympus, Tokyo, Japan) (Figure 1). First, we applied a long clip (HX-600-090L; Olympus) to the base of the stalk, close to the colonic wall, in order to prevent polypectomy-associated bleeding. The polyp stalk was then cut about $8 \mathrm{~mm}$ above the clip using the insulated-tip diathermy knife (Figure 2). Pure cutting current was used. No hemorrhage, perforation, or other complication occurred. Histological evaluation of the resection specimen revealed a serrated adenoma.

It is often difficult to remove large pedunculated colorectal polyps by endoscopic polypectomy using standard snare techniques [1,2]. Our approach was to perform an endoscopic resection using an insulated-tip diathermy knife which can be passed through the endoscope's ordinary working channel. This knife was devel- oped in Japan for endoscopic mucosal resection of early gastric cancer $[3,4]$. It has a ceramic ball at its tip which prevents electrosurgical perforation by the needleknife tip. Using this knife, it was safe and easy to cut the stalk of the polyp. Clipping the basal side of the stalk prior to cutting was vital for preventing polypectomy-induced bleeding. To our knowledge, this is the first report of endoscopic polypectomy using an insulated-tip diathermy knife. We believe this technique has the potential to become the method of choice for removal of pedunculated colorectal polyps when procedures involving snaring of the polyp head are not feasible. 

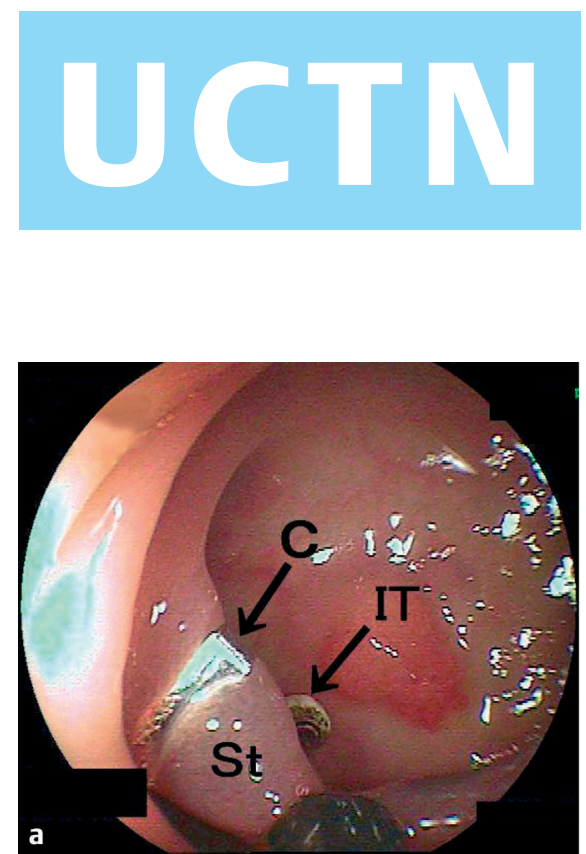

K. Akahoshi', M. Kubokawa',

T. Fujimaru', M. Matsumoto',

M. Kimura' ${ }^{1}$, H. Akiho', Y. Sumida',

M. Oya ${ }^{2}$

${ }^{1}$ Department of Gastroenterology,

Aso lizuka Hospital, lizuka, Japan

${ }^{2}$ Department of Pathology, Aso lizuka

Hospital, lizuka, Japan.

\section{References}

${ }^{1}$ Akahoshi K, Kojima H, Fujimaru Tet al. Grasping forceps-assisted endoscopic resection of large pedunculated gastrointestinal polypoid lesions. Gastrointest Endosc 1999; 50: 95 - 98

${ }^{2}$ Cipolletta L, Bianco MA, Rotondano Get al. Endoclip-assisted resection of large pedunculated colon polyps. Gastrointest Endosc 1999; 50: 405-406

${ }^{3}$ Ono H, Kondo H, Gotoda Tet al. Endoscopic mucosal resection for treatment of early gastric cancer. Gut 2001; 48: $225-229$

${ }^{4}$ Ohkuwa M, Hosokawa K, Boku Net al. New endoscopic treatment for intramucosal gastric tumors using an insulatedtip diathermic knife. Endoscopy 2001; 33: $221-226$

\section{Corresponding Author}

\section{K. Akahoshi, M.D.}

Department of Gastroenterology, Aso lizuka Hospital 3-83 Yoshio Iizuka 820-8505

Japan

Fax: $\quad$ +81-948-29-8747

E-mail: kakahoshi2@aol.com
Figure 2 Endoscopic view of the resection of a colorectal polyp using an insulated-tip diathermy knife. a The knife (IT) is used to cut the stalk (St) $8 \mathrm{~mm}$ above the clip (C). b The polyp is removed without bleeding. 